\title{
Two splice variants of the IDD14 transcription factor competitively form nonfunctional heterodimers which may regulate starch metabolism
}

Pil Joon Seo', Mi Jung Kim¹, Jae-Yong Ryu', Eun-Young Jeong' \& Chung-Mo Park¹,2

Alternative splicing of primary gene transcripts provides eukaryotic cells, with a critical scheme for enriching transcriptome and proteome diversity. Here we report that alternative splicing of the Arabidopsis INDERMINATE DOMAIN 14 (IDD14) transcription factor gene generates a competitive inhibitor in regulating starch metabolism. An alternatively spliced IDD14 form (IDD14 $\beta$ ), which is produced predominantly under cold conditions, lacks functional DNAbinding domain but is able to form heterodimers with the functional IDD14 form (IDD14 $\alpha$ ). IDD14 $\alpha$-IDD14 $\beta$ heterodimers have reduced binding activity to the promoter of Qua-Quine Starch (QQS) gene that regulates starch accumulation. Transgenic Arabidopsis plants overproducing IDD14 $\alpha$ (35S:IDD14 $\alpha$ ) exhibited retarded growth with pale green leaves as appeared on QQS-overexpressing plants. Notably, IDD14 $\beta$ overproduction rescued the 35S:IDD14 $\alpha$ phenotypes. We propose that alternative splicing of the IDD14 gene generates a self-controlled regulatory loop that may modulate starch accumulation in response to cold. 
T he regulation of transcription factors by dynamic formation of dimers and protein complexes is an important component of gene regulatory networks that provides a way of diversifying the affinity and specificity of protein-DNA interactions ${ }^{1}$. It also generates a wide variety of DNA-binding protein complexes from a limited number of transcription factors and regulatory proteins in eukaryotic genomes.

An illustrative example is a feedback regulatory module provided by competitive inhibitors that form nonfunctional heterodimers with specific transcription factors. Several competitive inhibitors have been reported in both animals ${ }^{2}$ and plants $s^{3,4}$. The inhibitor of DNA-binding (ID) proteins have sequence similarities to a subset of basic helix-loop-helix (bHLH) transcription factors that are involved in cell proliferation and differentiation in animals ${ }^{2}$. However, because these inhibitor proteins lack the basic region required for DNA binding, they prevent bHLH transcription factors from binding to DNA by forming heterodimers that possess reduced affinity for substrate DNA ${ }^{2}$.

A few competitive inhibitors have been characterized in plants in recent years. Similar to the structural organization of the ID proteins, the Little Zipper (ZPR) proteins consisting of 67-105 residues possess leucine zipper motifs that have limited sequence similarities to class III homeodomain-leucine zipper (HD-ZIP III) transcription factors functioning in shoot apical meristem formation and lateral organ development in Arabidopsis ${ }^{3,4}$. Although the ZPR proteins lack DNA-binding domains, they are able to competitively interact with HD-ZIP III transcription factors to form nonfunctional heterodimers. The MINI ZINC FINGER (MIF) proteins consisting of $\sim 100$ residues also function in a similar manner ${ }^{5}$. They have zinc finger (ZF) motifs that mediate protein-protein interactions. However, they lack other protein motifs normally present in the zinc finger-homeodomain (ZHD) transcription factors that regulate leaf development and floral architecture. It has been shown that the MIF1 protein reduces the DNA-binding affinity of the ZHD5 transcription factor by forming MIF1-ZHD5 heterodimers ${ }^{6}$. Although it has not been proven yet, the KIDARI (KDR) proteins have been suggested to act as a negative regulator of some bHLH transcription factors, such as LONG HYPOCOTYL IN FAR-RED1, in photomorphogenesis?

Alternative splicing is a biochemical process by which the exons of a primary gene transcript are reconstituted in multiple ways. It allows production of multiple proteins from a single gene, extending the proteomic and functional diversity in eukaryotes. However, in some cases, alternatively spliced isoforms apparently lack protein domains required for protein activities, obscuring the necessity of alternative splicing. For example, the Arabidopsis FCA gene encodes an RNA-binding protein that promotes flowering. The primary FCA gene transcript is alternatively spliced to yield four transcripts: $\alpha, \beta, \gamma$ and $\delta$. Among the four transcripts, only the $\gamma$ transcript is considered to encode the putative full-size FCA protein ${ }^{8}$.

In this work, we found that alternative splicing of a transcription factor gene serves as an active way of controlling the full-size transcription factor by producing a competitive inhibitor. Through genome-wide searches for alternatively spliced transcription factors in Arabidopsis, we identified a gene encoding the INDERMINATE DOMAIN 14 (IDD14) transcription factor. Alternative splicing of the IDD14 gene was induced under cold conditions, producing a spliced isoform IDD14 $\beta$ that lacks a functional DNA-binding domain. The IDD14 $\beta$ isoform attenuated the activity of the full-size IDD14 $\alpha$ transcription factor by competitively forming nonfunctional heterodimers. The IDD1 $4 \alpha$ transcription factor regulates starch metabolism by binding directly to the promoter of the QQS gene. The IDD14 $\beta$ form prevented the IDD14 $\alpha$ transcription factor from binding to the QQS gene promoter, providing a distinct role of alternative splicing in gene expression regulation.

\section{Results}

Alternative splicing of IDD14 gene. Protein structural analysis of plant transcription factors and their alternatively spliced isoforms suggest that at least some of the alternatively spliced isoforms lacking functional DNA-binding domains could act as competitive inhibitors. Recent studies have shown that a few alternatively spliced isoforms of animal transcription factors possess dominant negative activities ${ }^{9,10}$, supporting this hypothesis.

We compared transcription factor genes with alternatively spliced genes in Arabidopsis and rice deposited in public databases. The in silico analysis predicted that $\sim 340$ transcription factor genes undergo alternative splicing (Supplementary Data 1). Among these, we chose Arabidopsis IDD14 gene for further analysis, as an alternatively spliced IDD14 isoform fit the structural rules defined by known competitive inhibitors.

Comparing the two IDD14 cDNA sequences (designated $I D D 14 \alpha$ and $I D D 14 \beta$ ) with genomic sequence revealed that the first intron was retained in the IDD14 $\beta$ cDNA (Fig. 1a). Reverse transcription PCR (RT-PCR) using cDNA-specific primers detected both transcripts (Fig. 1b). Whereas IDD $14 \alpha$ contains three copies of ZF motifs, IDD14 $\beta$ lacks the ZF1 motif (Fig. 1c and Supplementary Fig. S1).

We produced transgenic plants overexpressing either the $I D D 14 \alpha$ or the IDD14 $\beta$ gene. We also obtained an IDD14-deficient idd14-1 mutant (Supplementary Fig. S2). The IDD14 $\alpha$-overexpressing plants exhibited retarded growth with pale green leaves showing some effects of gene dosage (Supplementary Fig. S2). The 35S:IDD14 $\alpha-3$ transgenic line, which has a mild phenotype, was used in subsequent assays (hereafter referred to as $35 \mathrm{~S}: I D D 14 \alpha$ ). Whereas the levels of $I D D 14 \alpha$ and $I D D 14 \beta$ transcripts were similar in the $35 \mathrm{~S}: I D D 14 \alpha$ and $35 \mathrm{~S}: I D D 14 \beta$ transgenic plants, respectively (Fig. 1d), the 35S:IDD14 $\beta$ phenotypes were similar to those of the idd14-1 mutant in that both exhibited accelerated growth with slightly early flowering (Fig. 1e and Supplementary Fig. S3).

Attenuation of IDD14 $\alpha$ activity by IDD14 $\beta$. We hypothesized that the $35 \mathrm{~S}: I D D 14 \beta$ phenotypes would be due to the negative activity of IDD14 $\beta$, possibly by forming heterodimers with IDD14 $\alpha$. IDD14 $\alpha$-IDD14 $\beta$ interactions were therefore examined in yeast cells, and, as expected, IDD1 $\alpha \alpha$ and IDD14 $\beta$ formed heterodimers (Fig. 2a). In addition, as inferred from the structural organization, IDD14 $\alpha$ and IDD14 $\beta$ formed homodimers. Assays using a series of IDD $14 \alpha$ deletions revealed that the interactions occurred by the coiled-coil motif (residues 311-350; Fig. 2b). In vitro pulldown assays using a recombinant maltose-binding protein-IDD $14 \alpha$ (MBP-IDD14 $\alpha$ ) fusion and IDD14 $\alpha$ and IDD14 $\beta$ proteins, which were produced by in vitro translation, confirmed that IDD14 $\alpha$ forms homodimers (Fig. 2c) and heterodimers with IDD14 $\beta$ (Fig. 2d). Bimolecular fluorescence complementation (BiFC) assays in Arabidopsis protoplasts showed that IDD14 $\alpha$ and IDD14 $\beta$ were colocalized into the nucleus (Fig. 2e and Supplementary Fig. S4). We also obtained identical results from immunoprecipitation assays of protein extracts from Nicotiana benthamiana leaf cells expressing the MYC-IDD14 $\alpha$ and MYC-IDD $14 \beta$ gene fusions (Supplementary Fig. S5), further supporting the IDD $14 \alpha-I D D 14 \beta$ interactions.

Our observations suggest that IDD14 $\beta$ would attenuate the DNAbinding activity of IDD $14 \alpha$ homodimers by competitively forming heterodimers. To examine this, the $I D D 14 \beta$ gene was expressed under the control of a methionine (Met)-suppressible promoter in yeast cells expressing BD-IDD14 $\alpha$ and AD-IDD14 $\alpha$ fusions. $\beta$-Galactosidase ( $\beta$-Gal) activity assays showed that IDD14 $\beta$ induction substantially repressed the formation of IDD $14 \alpha$ homodimers (Fig. $3 a-c)$. To examine the negative activity of IDD14 $\beta$ in planta, we genetically crossed 35S:IDD14 $\alpha$ plants with $35 \mathrm{~S}$ : $I D D 14 \beta$ plants. As inferred from the suppression of IDD14 $\alpha$ homodimer formation by IDD14 $\beta$, 35S:IDD14 $\alpha$ phenotypes were rescued 


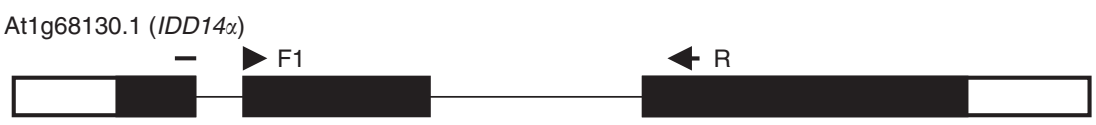

At1g68130.2 (IDD14ß)

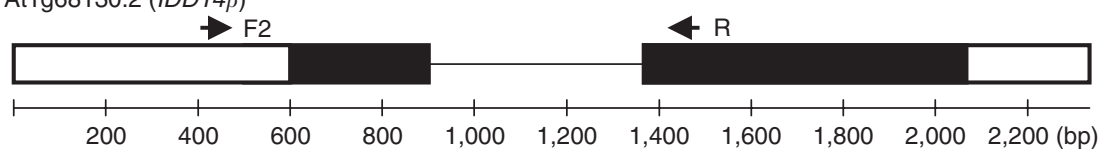

C

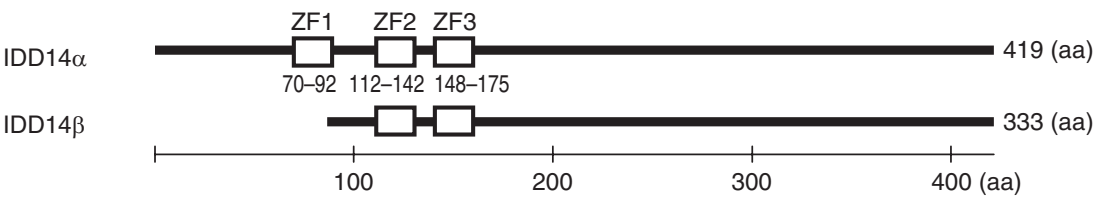

b

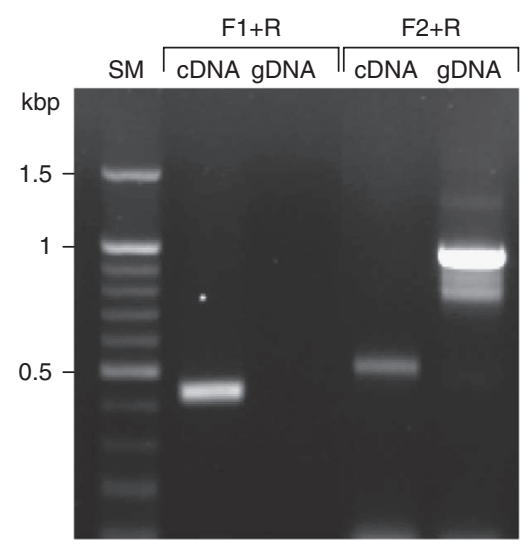

d

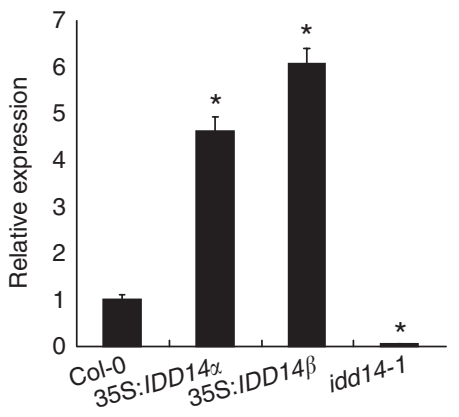

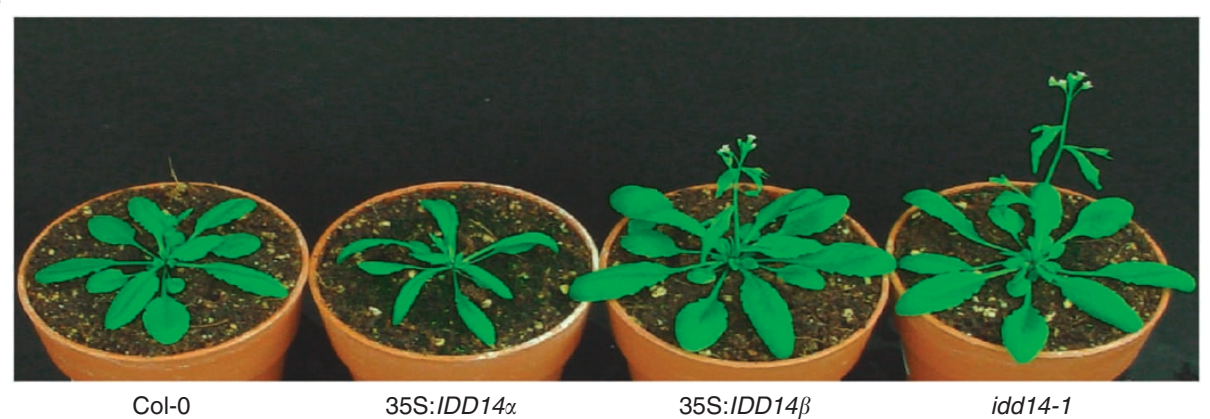

Figure 1 | Similar phenotypes of 35S:IDD14 $\beta$ transgenic and idd14-1 mutant plants. (a) Genomic structure of IDD14 and alternatively spliced variants. White boxes, untranslated regions; black boxes, exons; F1 and F2, forward primers; R, reverse primer. (b) Detection of alternatively spliced transcripts by RT-PCR and genomic PCR. SM, size marker. (c) Protein structure of IDD14 proteins. ZF, zinc finger. (d) IDD14 gene expression. Transcript levels were determined by qRT-PCR using total RNA samples extracted from 2-week-old whole plants. Biological triplicates were averaged and statistically analysed using a Student's t-test ( ${ }^{\star} P<0.01 ; 35 S: I D D 14 \alpha: P=0.0032, n=6 ; 35 S: I D D 14 \beta: P=0.0002, n=6 ;$ idd14-1: $\left.P=0.0005, n=6\right)$. Bars indicate standard error of the mean. (e) Phenotypes of 35S:IDD14 $\alpha, 35$ S:IDD14 $\beta$ and idd14-1 plants.

in the 35S:IDD14 $\alpha$ X 35S:IDD14 $\beta$ plants (Fig. 3d), demonstrating that IDD14 $\beta$ negatively regulates IDD $14 \alpha$ activity by inhibiting its homodimer formation.

However, the transcriptional activation activity of IDD $14 \alpha$ was unaffected by IDD14 $\beta$, as assayed by the transient expression assays in Arabidopsis protoplasts ${ }^{11}$ (Fig. 3e,f), suggesting that the negative activity of IDD14 $\beta$ is due to the inhibition of DNA binding.

Binding of IDD14 $\alpha$ to the QQS gene promoter. The 35S:IDD14 $\alpha$ phenotypes, such as growth retardation and pale green leaves with downward curling (Supplementary Fig. S2), are frequently observed in plants with disturbed sugar metabolism ${ }^{12,13}$, suggesting that the IDD14 gene may have metabolic roots. In support of this view, several IDD members have been implicated in sugar and starch metabolism in Arabidopsis and maize $\mathrm{e}^{14,15}$. To better understand the physiological role of IDD14, we examined the expression of an extensive set of genes that are involved in sugar and starch metabolism in 35S:IDD14 $\alpha$ and 35S:IDD14 $\beta$ transgenic and idd 14- 1 mutant plants. We found that only the QQS gene was significantly upregulated in 35S:IDD14 $\alpha$ transgenic plants (Fig. 4a and Supplementary Fig. S6). Notably, the QQS gene was downregulated in 35S:IDD14 $\beta$ transgenic plants, as occurred in idd14-1 mutant. However, QQS gene expression was recovered in the 35 S:IDD $14 \alpha \times 35$ S:IDD $14 \beta$ plants, suggesting that IDD $14 \alpha$ activity is correlated with the QQS gene.

The QQS gene negatively regulates starch accumulation ${ }^{16}$. Measurements of starch content revealed that it was detectably lower in $35 \mathrm{~S}: I D D 14 \alpha$ transgenic plants (Fig. $4 \mathrm{~b}$ ). In contrast, it was elevated by $\sim 8 \%$ in 35 S:IDD $14 \beta$ transgenic and idd14-1 mutant plants. Meanwhile, the starch content in 35S:IDD14 $\alpha \times 35$ S:IDD14 $\beta$ plants was similar to that in Col-0 plants, in agreement with QQS expression patterns.

To rule out the possibility that differential starch contents are caused by developmental defects owing to the constitutive expression of IDD14 genes, we produced transgenic plants expressing IDD $14 \alpha$ and IDD $14 \beta$ genes under a $\beta$-estradiol-inducible promoter and measured starch contents 3 days after induction. Whereas starch content was significantly reduced in IDD14 $\alpha$-expressing plants, it was largely uninfluenced in IDD14 $\beta$-expressing plants (Supplementary Fig. S7), supporting the linkage of the IDD14 transcription factor with starch accumulation and disturbed starch metabolism as the cause of the pale green appearance of 35S:IDD14 $\alpha$ transgenic plants.

To explore whether IDD14 transcription factors directly regulates the QQS gene, we fused a MYC-coding sequence in-frame to the $3^{\prime}$ ends of IDD14 $\alpha$ and IDD14 $\beta$ genes and transformed the expression construct into Arabidopsis plants. Chromatin immunoprecipitation assays were performed using 35S:IDD14 $\alpha-M Y C$ and 35S:IDD14 $\beta$ MYC transgenic plants. The results showed that IDD14 $\alpha$ bound to the BS2 sequence that covers residues -924 to -637 in the QQS gene promoter (Fig. 4c). In contrast, no discernible binding activity was detected with IDD14 $\beta$, showing that partial loss of the ZF domains in IDD14 $\beta$ disrupts its DNA-binding activity.

The functional relationship between IDD14 and the QQS gene was also supported by phenotypic analysis. Both 35S:IDD14 $\alpha$ and 35S:QQS transgenic plants showed similar phenotypes, such as retarded growth and downward leaf curling (Fig 4d,e), and low starch content (Fig. 4f). Furthermore, although starch content was higher in 35S:IDD14 $\beta$ transgenic plants, it was significantly reduced 
a
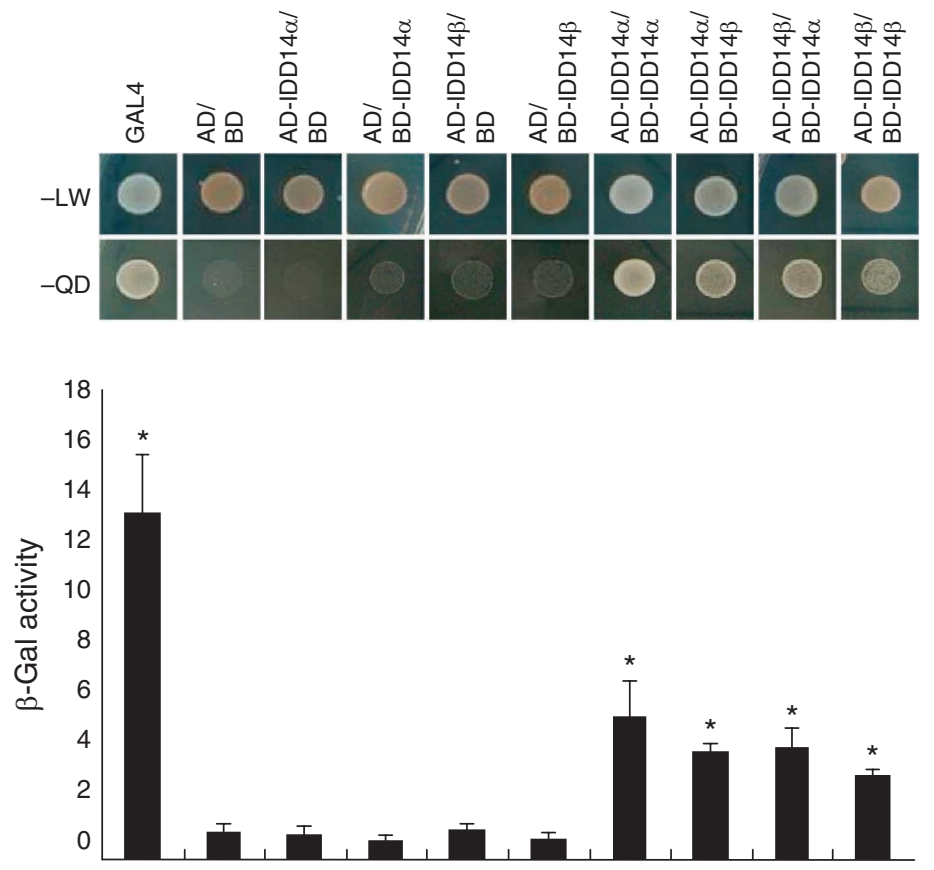

C
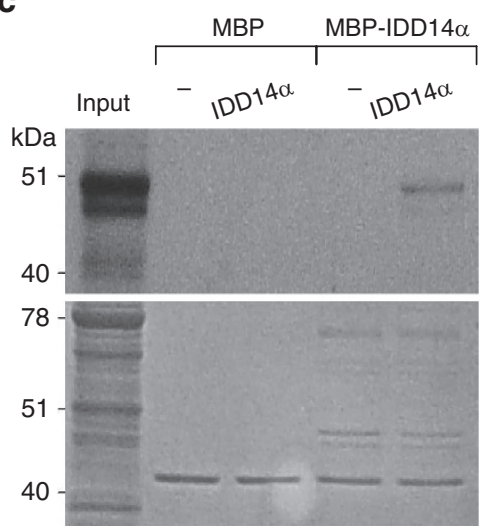

d
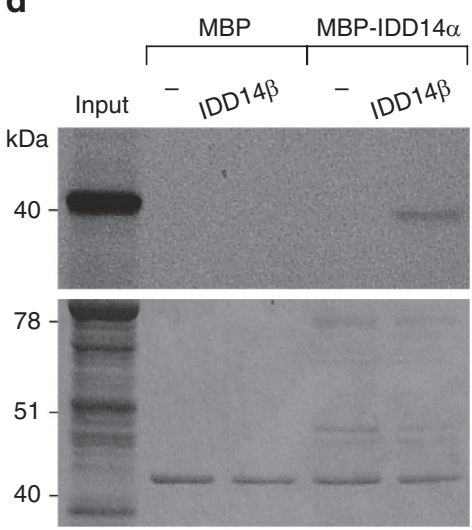

b
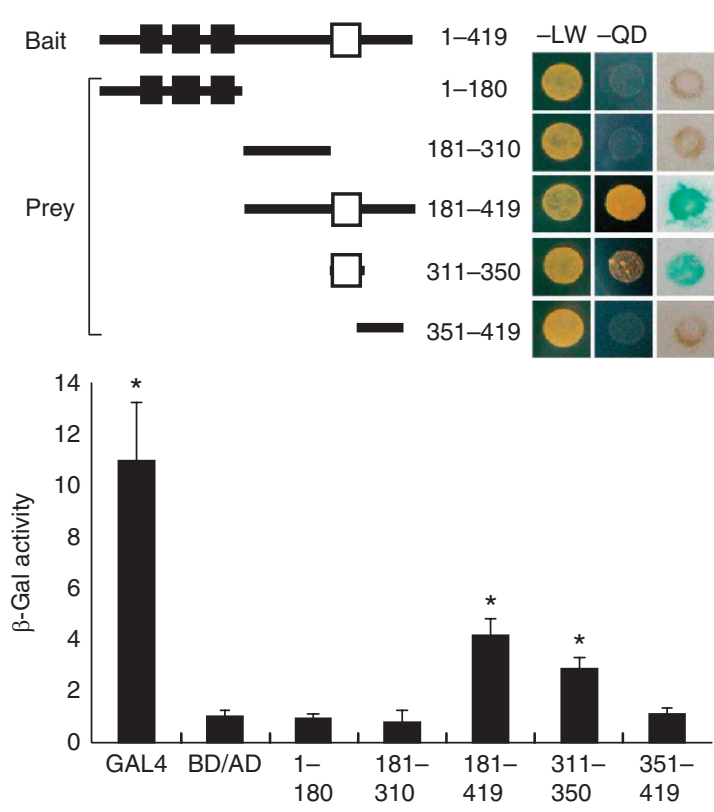

e

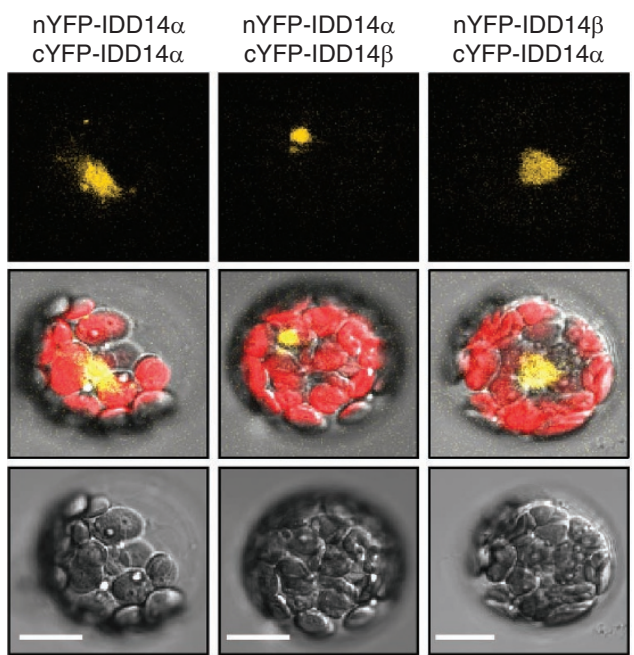

Figure 2 | IDD14 $\alpha$-IDD14 $\beta$ interactions. In $\mathbf{a}$ and $\mathbf{b}$, measurements of $\beta$-Gal activities were averaged and statistically treated (bottom panels). Bars indicate standard error of the mean. (a) Yeast coexpression assays. Cell growth on selective media without Leu, Trp, His and Ade (-QD) indicates positive interactions. $\beta$-Gal activities were normalized by dividing total activity by optical cell density ( $t$-test, ${ }^{\star} P<0.01 ; G A L 4: P=1.2 \times 10^{-7}, n=9$;

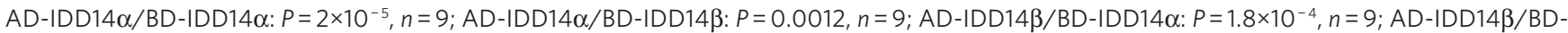
IDD14 $\beta$ : $P=0.0058, n=9)$. (b) Mapping of interacting domain. Interactions were examined both by cell growth on selective media (-QD) and by colony filter-lift assays (top panel; $t$-test, ${ }^{\star} P<0.01 ;$ GAL4: $P=1.9 \times 10^{-9}, n=9 ; 181-419: P=0.0002, n=9 ; 311-350: P=0.0033, n=9$ ). White box, coiled-coil motif. (c, d) In vitro pull-down assays. A recombinant MBP-IDD14 $\alpha$ fusion and in vitro translated radio-labelled IDD14 $\alpha$ (c) and IDD14 $\beta$ (d) were used. Bottom panels were parts of Coomassie blue-stained gels. (e) BiFC assays. Partial YFP fusion constructs were transiently expressed in Arabidopsis protoplasts. Scale bar, $20 \mu \mathrm{m}$.

in the genetic cross of 35S:IDD14 $\beta$ and 35S:QQS transgenic plants (Supplementary Fig. S8), confirming that QQS functions downstream of the IDD $14 \alpha$ transcription factor.

Cold-induced IDD14 alternative splicing in starch metabolism. A critical question that remained was how IDD14 alternative splicing was induced. We examined the effects of growth hormones, sugars and various stresses on the relative ratio of $I D D 14 \alpha$ and IDD14 $\beta$ transcripts. Interestingly, IDD14 alternative splicing was greatly influenced only by cold $\left(4^{\circ} \mathrm{C}\right.$; Fig. $\left.5 \mathrm{a}\right)$. Whereas the level of IDD $14 \alpha$ transcript was unchanged, the level of IDD14 $\beta$ transcript was gradually elevated in response to cold. In contrast, the level of QQS transcript steadily decreased, consistent with the notion that
IDD14 $\beta$ negatively regulates the activity of IDD $14 \alpha$ that activates the QQS gene. The QQS gene was not influenced by other environmental signals and sugars (Supplementary Fig. S9).

We next examined the effects of cold on the accumulation of starch. Plants were exposed to cold at zeitgeber time 0 (ZT0), and relative starch contents were measured at ZT0 and ZT24. Starch contents were low at ZT0 and ZT24 in plants incubated at $23^{\circ} \mathrm{C}$ (Fig. 5b), as has been reported previously ${ }^{16,17}$. Starch accumulated rapidly during the light period but degraded during the dark period. In contrast, under cold conditions, starch contents increased approximately fourfold in 35S:IDD14 $\beta$ transgenic and idd14-1 mutant plants as they did in Col-0 plants at ZT24, which is consistent with the suppression of the QQS gene by cold (Supplementary Fig. S10). 
a

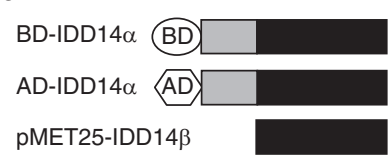

b
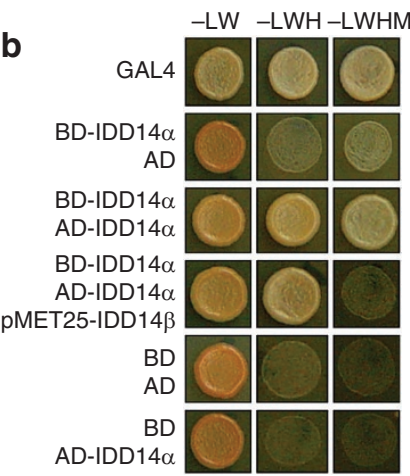

AD-IDD14a

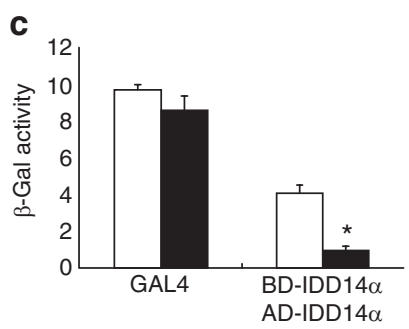

d Col-0 35S:IDD $14 \alpha \times$ Col-0

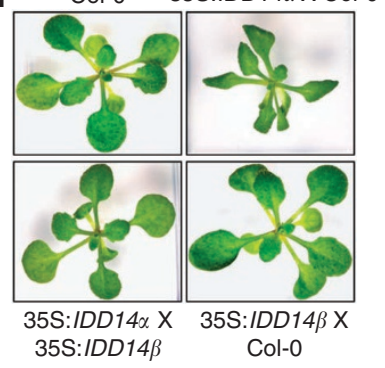

e

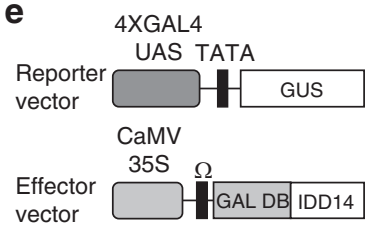

UAS: Upstream activation seq $\Omega$ : Translational enhancer DB: DNA-binding domain

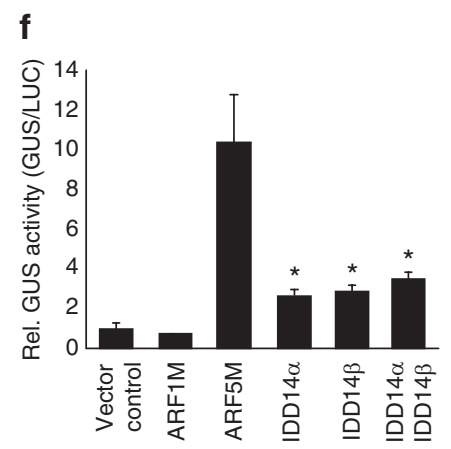

Figure 3 | Competitive inhibition of IDD14 $\alpha$ activity by IDD14 $\beta$. (a) Constructs used for yeast three-hybrid assays. The IDD14 $\beta$ gene was expressed under the control of the methionine (Met)-suppressible promoter (pMET25-IDD14 $\beta$ ). AD, activation domain; BD, DNA-binding domain. (b) Inhibition of IDD14 $\alpha$ dimer formation by IDD14 $\beta$. The IDD14 $\beta$ gene is not expressed on selective media without Leu, Trp and His (-LWH), but expressed on selective media without Leu, Trp, His and Met (-LWHM). (c) Quantification of yeast coexpression assays. $\beta$-Gal activities were measured in the presence (white bars) or absence (black bars) of Met ( $t$-test, $\left.{ }^{\star} P=0.0013, n=12\right)$. (d) Suppression of 35S:IDD14 $\alpha$ phenotypes by IDD14 $\beta$ coexpression. (e) Reporter and effector vectors used for transient expression assays in Arabidopsis protoplasts. GAL4 transient expression assays were carried out as described previously"1. The Renilla luciferase gene served as an internal control to normalize values in individual assays. (f) Transcriptional activation activity of IDD14 isoforms. Five independent measurements were averaged. Bars indicate standard error of the mean ( $t$ test, ${ }^{\star} P<0.01$; IDD14 $\alpha: P=0.0042, n=15 ;$ IDD14 $\beta: P=0.001, n=15 ;$ IDD14 $\alpha$ IDD14 $\beta: P=3.2 \times 10^{-6}, n=15$ ).

However, the degree of elevation was reduced in 35S:IDD14 $\alpha$ transgenic plants, probably because of the increased QQS activity. These observations indicate that starch degradation is reduced during the dark period in response to cold.

The effects of IDD14 $\beta$ on the expression of the QQS gene in response to cold was verified by examining levels of QQS transcript in 35S:IDD14 $\alpha$ transgenic plants and in the idd14-1 mutant overexpressing the IDD14 $\alpha$ gene (idd14-1:IDD14 $\alpha$ ). The level of QQS transcript was reduced by $\sim 45 \%$ in 35 S:IDD $14 \alpha$ transgenic plants as it was in Col-0 plants exposed to cold (Fig. $5 \mathrm{c}$ ). However, it was reduced by $25 \%$ in idd14-1:IDD $14 \alpha$ plants. These data are at least, in part, explained by the negative regulation of IDD $14 \alpha$ activity by IDD14 $\beta$. Measurements of starch contents also
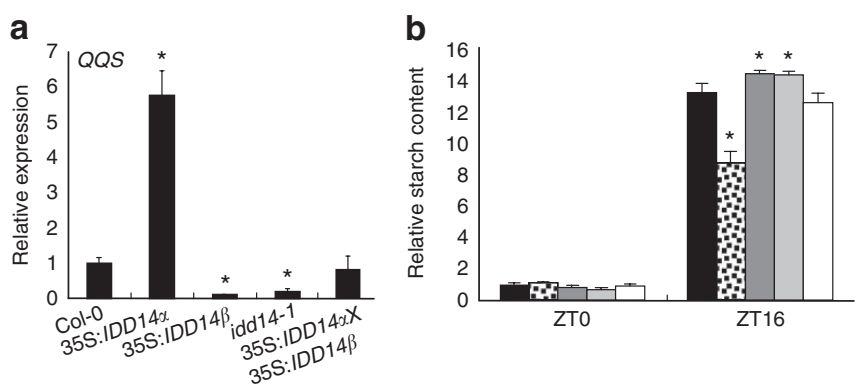

C
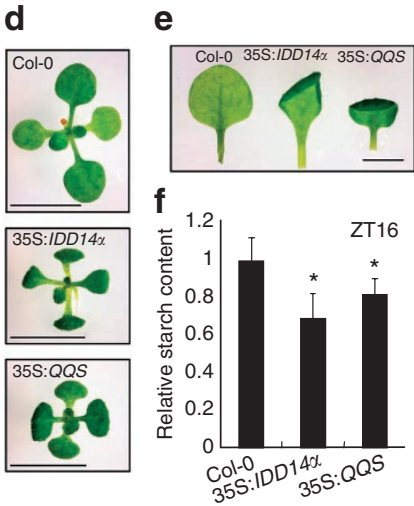

Figure 4 | Regulation of the QQS gene by IDD14 proteins. (a) QQS gene expression. Transcript levels were determined by qRT-PCR. Biological triplicates were averaged and statistically treated ( $t$-test, ${ }^{\star} P<0.01$; 35S:IDD14 $\alpha: P=2.2 \times 10^{-8}, n=6 ; 35 S: I D D 14 \beta: P=0.0001, n=6$;

idd14-1: $P=5 \times 10^{-5}, n=6$ ). Bars indicate standard error of the mean. (b) Measurements of starch contents. The value 1 is equal to $0.3 \mathrm{mg}$ per $\mathrm{g}$ fresh weight. Bars indicate standard error of the mean. Black bars, Col-0; dotted bars, 35S:IDD14 $\alpha$; dark grey bars, 35S:IDD14 $\beta$; light grey bars, idd14-1; white bars, 35S:IDD14 $\alpha \times 35$ S:IDD14 $\beta$ ( $t$-test, ${ }^{*} P<0.01 ; 35 S: I D D 14 \alpha$ : $P=1.2 \times 10^{-6}, n=12 ; 35 \mathrm{~S}:$ IDD14 $\beta: P=0.005, n=12 ;$ idd14-1: $P=0.0061, n=12$ ). (c) Chromatin immunoprecipitation assays. BS1, BS2 and BS3 are putative binding sites in the QQS gene promoter (top panel). Three-week-old plants were used. Three measurements were averaged and statistically treated (bottom panel; $t$-test, ${ }^{\star} P=0.0076, n=9$ ). Bars indicate standard error of the mean. White bars, Col-0; black bars, 35S:IDD14 $\alpha$-MYC; grey bars, 35S:IDD14 $\beta$-MYC. (d) Phenotypes of 35S:IDD14 $\alpha$ and 35S:QQS transgenic plants. Scale bar, $5 \mathrm{~mm}$. (e) Leaf morphology. Scale bar, $2 \mathrm{~mm}$. In $\mathbf{d}$ and e, 10-day-old plants were photographed. (f) Starch contents of 35S:IDD14 $\alpha$ and 35S:QQS transgenic plants. The value 1 is equal to $2.2 \mathrm{mg}$ per $\mathrm{g}$ fresh weight. Bars indicate standard error of the mean ( $t$-test, ${ }^{\star} P<0.01$; 35S:IDD14 $\left.\alpha: P=1.6 \times 10^{-6}, n=18 ; 35 \mathrm{~S}: \mathrm{QQS}: P=0.002, n=18\right)$. $\ln \mathbf{b}$ and $\mathbf{f}$, five measurements were averaged and statistically analysed. Bars indicate standard error of the mean. ZT, zeitgeber time.

support the role of IDD14 $\beta$ on the expression of QQS gene in regulating starch accumulation. Starch contents were lower in idd14-1:IDD14 $\alpha$ plants than in 35S:IDD14 $\alpha$ transgenic plants under cold (Fig. 5d).

To explore the biological meaning of the starch accumulation with regard to temperature, patterns of starch accumulation were monitored throughout the ZT times after exposure to cold. Starch still accumulated during the light period but to a lesser degree than in plants incubated at $23^{\circ} \mathrm{C}$ (Fig. 5e). After ZT16, whereas starch content rapidly decreased in plants grown at $23^{\circ} \mathrm{C}$, it was only slightly reduced in plants exposed to cold, which is in agreement with the suppression of the QQS gene that occurs in plants under cold conditions (Fig. 5e). Together, these observations indicate that the regulation of the QQS gene by IDD14 alternative splicing is physiologically important during the dark period and when plants are exposed to cold (Fig. 5f). 


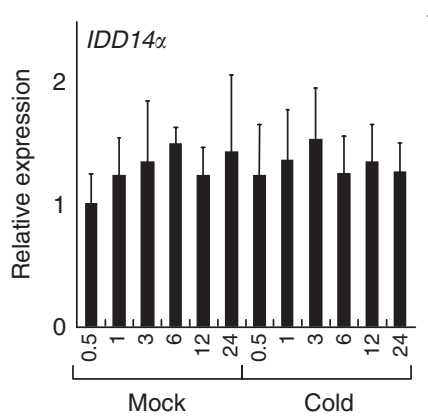

C

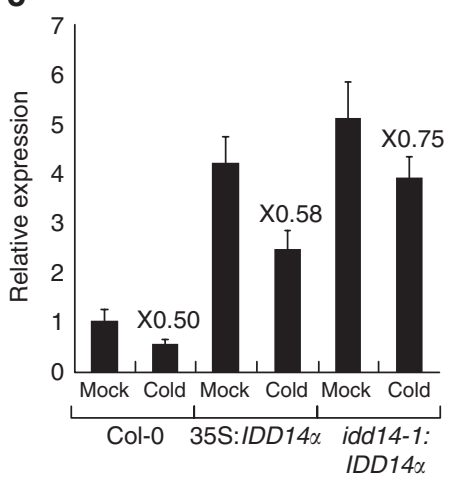

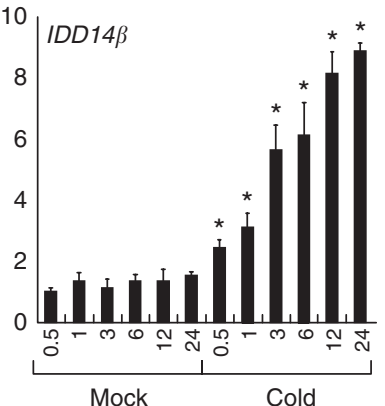

d

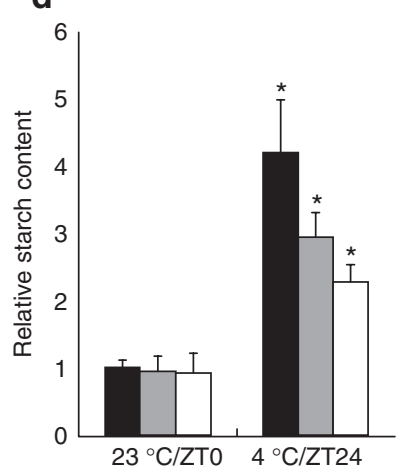

b

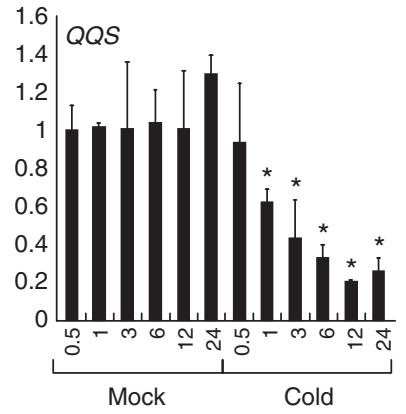

e
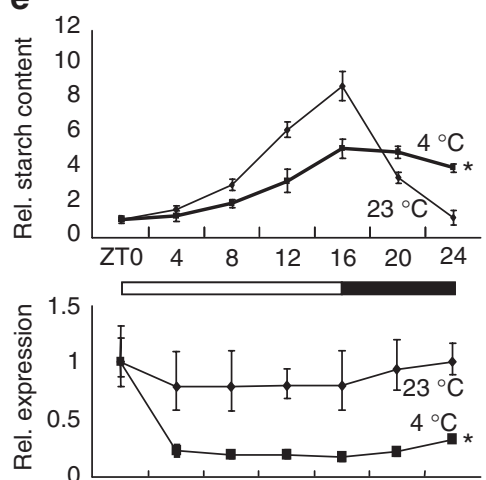

f

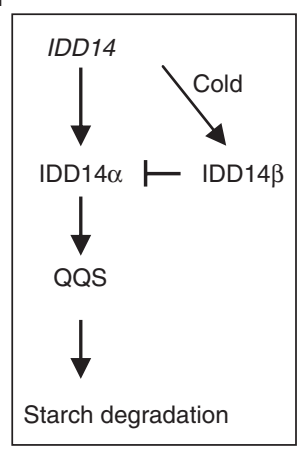

Figure 5 | Cold induction of IDD14 alternative splicing. (a) Effects of cold on IDD14 and QQS gene expression ( $t$-test, ${ }^{\star} P<0.01, n=6$ ). Two-week-old plants were treated with cold. (b) Effects of cold on starch accumulation. Black bars, Col-0; dark grey bars, 35S:IDD14 $\alpha$; light grey bars, 35S:IDD14 $\beta$; white bars, idd14-1 (t-test, $\left.{ }^{\star} P=0.0033, n=15\right)$. (c) Effects of cold on QQS gene expression. Two-week-old plants were treated with cold. (d) Measurements of starch contents. Black bars, Col-0; grey bars, 35S:IDD14 $\alpha$; white bars, idd14-1:IDD14 $\alpha$ (t-test, ${ }^{*} P<0.01$; Col-0: $P=3.1 \times 10^{-8}, n=15 ; 35 \mathrm{~S}: I D D 14 \alpha: P=0.0002$, $n=15$; idd14-1:IDD14 $\alpha: P=0.0015, n=15$ ). In $\mathbf{b}$ and $\mathbf{d}$, cold treatment was initiated at ZTO. (e) Kinetic measurements of starch contents (top panel) and QQS gene expression (bottom panel). Plants at ZTO were exposed to cold ( $t$-test, $\left.{ }^{\star} P<0.01, n=15\right)$. (f) Working model of the IDD14-mediated regulatory module in starch accumulation under cold conditions. In $\mathbf{a}, \mathbf{c}$ and $\mathbf{e}$, transcript levels were determined by qRT-PCR. Whole plants were used for total RNA extraction. Biological triplicates were averaged and statistically treated. In $\mathbf{b}, \mathbf{d}$ and $\mathbf{e}$, shoots of two-week-old plants grown on Murashige and Skoog agar plates were used for starch extraction. Five measurements were averaged and statistically treated. The value 1 is equal to $0.3 \mathrm{mg}$ per $\mathrm{g}$ fresh weight.

\section{Discussion}

RNA splicing is a post-transcriptional event that excises intron sequences from primary RNA transcripts and serves as a gene regulatory step in many eukaryotic genes. Alternative splicing generates multiple mRNAs from a single genetic locus through alternative selection of splice sites in the primary RNA transcripts, extending structural and functional diversities from the limited number of genes in the genome ${ }^{8}$. Alternative splicing of transcription factor genes has rarely been documented in plants, mostly because of nonfunctionality of the truncated forms.

In this work, we demonstrated that an alternative spliced IDD14 isoform (IDD14 $\beta$ ) has a functional role in starch metabolism under cold conditions. The IDD14 transcription factor funcitons as homodimers in regulating target genes. The IDD14 $\beta$ isoform lacks functional DNA-binding domain and thus is unable to bind to DNA. However, as it possesses intact protein-protein interaction domain, it can form heterodimers with the functional IDD $14 \alpha$ form. The heterodimers has reduced DNA-binding activity. Therefore, the IDD14 $\beta$ isoform is functionally very similar to the competitive peptide inhibitors, such as ZPR3 (refs 3,4). It is envisaged that competitive inhibition of transcription factors by truncated isoforms provides a versatile regulatory scheme in gene expression regulation under stress conditions. Subtle changes in the ratio of intact transcription factors and alternatively spliced variants would profoundly affect expression of target genes. It seems that alternative splicing, in coordination with gene transcriptional control, may help to maintain the appropriate level of transcription factor activities under a given growth conditions. Furthermore, it is also envisioned that alternative splicing of transcription factors guarantees prompt transcriptional responses to environmental fluctuations.

Several more competitive peptide inhibitors, in addition to ZPR proteins, have been identified recently in plants as well as in animals. Interestingly, phylogenetic analyses of genes encoding the competitive inhibitors and their target transcription factors in representative land plants, suggest that they are derived from functional transcription factor by deletion of functional domains before diversification of seed plants ${ }^{3}$. For example, although ZHD transcription factors are conserved in many land plants, including angiosperms, gymnosperms, seedless vascular plant (Selaginella) and nonvascular plant (moss), their competitive inhibitors MIFs are found only in seed plants, such as Arabidopsis, rice and cotton, supporting the recent occurrence of competitive inhibitors ${ }^{6}$. Similarly, ZPR proteins are present in Arabidopsis, rice and maize, but not in other plant databases ${ }^{3}$.

The IDD14 transcription factor is regulated by a competitive inhibitor that is produced by alternative splicing. Comparative genomic analysis showed that it is conserved in diverse plant species (Supplementary Table S1). So far, alternative splicing of the IDD14 gene was demonstrated only in Arabidopsis and has been predicted in rice (http://www.plantgdb.org). It is likely that competitive inhibition of transcription factors by alternatively spliced variants has a recent evolutionary origin.

Starch accumulation is closely linked to plant responses to cold stress. Soluble sugars accumulate during the light period under 
cold conditions, and controlled starch degradation has a role in this process $^{18-22}$. Our data strongly support that alternative splicing of IDD14 modulates its role in regulating starch metabolism under cold conditions. The IDD14 $\alpha$ form regulates directly the QQS gene that induces starch degradation ${ }^{16}$. The QQS gene is repressed by cold via the self-regulatory module provided by cold-induced alternative splicing of IDD14. On the basis of previous observations, as well as our own, we propose that the competitive inhibition of IDD14 $\alpha$ activity by an alternatively spliced variant IDD $14 \beta$ would serve as a cold adaptation strategy, helping a plant maintain a certain amount of starch during the dark period; this starch may be required to withstand cold conditions during the light period.

We found that QQS gene has a role in IDD14 regulation of starch metabolism in Arabidopsis, in which an alternatively spliced variant acts as a self-regulatory inhibitor. Molecular genetic analysis also supports the negative role of QQS gene in starch accumulation. However, it is premature to generalize our findings. No QQS gene homologues have been characterized in other plant species yet. Although the QQS gene acts as a negative regulator of starch accumulation ${ }^{16}$, it is unknown how it influences starch metabolism. More studies are required to see whether the IDD14 regulation of QQS gene is also applicable to other plant species. It will also be worthy to explore the biochemical role of QQS protein in regulating sugar metabolism. In addition, broader searches for the targets of IDD14 transcription factor, in addition to QQS, would be helpful in resolving the uncertainties.

\section{Methods}

Plant materials and growth conditions. All Arabidopsis thaliana lines used were in the Col-0 background. Plants were grown in a controlled culture room at $22^{\circ} \mathrm{C}$ with a relative humidity of $55 \%$ under long days (16-h light/8-h dark) with white light illumination $\left(120 \mu \mathrm{mol}\right.$ photons per $\left.\mathrm{m}^{2} \mathrm{~s}\right)$ provided by fluorescent FLR40D/A tubes (Osram)

The idd14-1 mutant (GABI_655E05) was isolated from a mutant pool of T-DNA insertion lines deposited in the Nottingham Arabidopsis Stock Centre (University of Nottingham, UK). Homozygotic lines were obtained by herbicide selection for three or more generations and by analysis of segregation ratios.

Analysis of transcript levels. Quantitative real-time RT-PCR (qRT-PCR) was used to measure transcript levels. RNA sample preparation, reverse transcription and quantitative-PCR were carried out based on the rules that have recently been proposed ${ }^{23}$ to ensure reproducible and accurate measurements. Extraction of total RNA samples from appropriate plant materials and RT-PCR conditions have been described previously ${ }^{24}$. The RNA samples were pretreated extensively with an RNAse-free DNAse to eliminate any contaminating genomic DNA before use.

qRT-PCR was carried out in 96-well blocks using an Applied Biosystems 7500 Real-Time PCR System (Applied Biosystems) using the SYBR Green I master mix in a volume of $25 \mu \mathrm{l}$. The PCR primers were designed using the Primer Express Software installed into the System and listed in Supplementary Table S2. The twostep thermal cycling profile used was $15 \mathrm{~s}$ at $94^{\circ} \mathrm{C}$ and $1 \mathrm{~min}$ at $68^{\circ} \mathrm{C}$. An $e I F 4 \mathrm{~A}$ gene (At3g13920) was included in the reactions as an internal control for normalizing the variations in cDNA amounts used ${ }^{25}$. All qRT-PCR reactions were carried out in biological triplicates using RNA samples extracted from three independent plant materials grown under identical growth conditions. The comparative $\Delta \Delta C_{\mathrm{T}}$ method was used to evaluate relative quantities of each amplified product in the samples. The threshold cycle $\left(C_{\mathrm{T}}\right)$ was automatically determined for each reaction by the System set with default parameters. The specificity of the PCR reactions was determined by melt curve analysis of the amplified products using the standard method installed in the System.

Subcellular localization of IDD14 proteins. For detection by fluorescence microscopy, the red fluorescent protein (RFP)- and green fluorescent protein (GFP)coding sequences were fused in-frame to the $3^{\prime}$ ends of the IDD14 $\alpha$ and IDD14 $\beta$ sequences, respectively, and the fusions were subcloned into the p326-RFP vector for RFP fusion and the pB7FWG2 vector for GFP fusion. The expression constructs were transformed into Arabidopsis protoplasts by polyethylene glycol-calcium transfection $^{26}$. The subcellular distribution of the IDD14 proteins was visualized by differential interference contrast (DIC) microscopy and fluorescence microscopy. The GFP and RFP fusion proteins were excited at 488 and $568 \mathrm{~nm}$, and the green and red fluorescence signals were filtered with HQ515/30 and HQ600/50 emission filters, respectively. The auto-fluorescence of chlorophylls was excited at $568 \mathrm{~nm}$ and emitted with the E600LP filter. The merged signals were obtained using a Confocal Assistant 4.02 (Todd Clark Brelje, Freeware).
Yeast two-hybrid assays. Yeast two-hybrid assays were performed using the BD Matchmaker system (Clontech). The pGADT7 vector was used for GAL4 AD (activation domain), and the pGBKT7 vector was used for GAL4 BD (DNA-binding domain). The yeast strain AH109 (Leu-, Trp-, Ade-, His-), which has chromosomally integrated reporter genes lacZ and HIS under the control of the GAL1 promoter, was used for transformation. The PCR products were digested with NdeI and EcoRI and subcloned into the pGBKT7 and pGADT7 vectors. Transformation of the AH109 cells was performed according to the manufacturer's instructions. Colonies obtained were streaked on a medium without His, Ade, Leu and Trp. To confirm the results, $\beta$-Gal assays were carried out according to the system procedure. For colony filter-lift assays, yeast cells carrying different combinations of plasmids were grown on -LW plate and transferred to a Whatman no. 5 paper filter. Cells were permeabilized by freeze-thaw treatment of the filters. The filters carrying the cells were then placed over filters presoaked with Z-buffer-X-Gal solution according to the protocol of the Matchmaker system.

The pBridge vector (Clontech) was used for yeast three-hybrid screening. The IDD $14 \alpha$ cDNA was amplified by RT-PCR, and the PCR product was digested with $E c o$ RI and SalI and subcloned into the pBridge vector, resulting in the BD-IDD $14 \alpha$ construct. The IDD14 $\beta$ cDNA was subcloned into the NotI-digested pBridge vector so that its expression was controlled by the pMET25 promoter. The expression constructs (BD-IDD $14 \alpha$ and pMET25-IDD $14 \beta$ in the pBridge vector and $\mathrm{AD}-\mathrm{IDD} 14 \alpha$ in the pGADT7 vector) were cotransformed into the AH109 cells The colonies were streaked on media without Leu, Trp and His supplemented with or without methionine.

In vitro pull-down assays. Recombinant MBP and MBP-IDD $14 \alpha$ proteins were produced in Escherichia coli BL21-CodonPlus (DE3)-RIL strains (Stratagene) and purified as follows. One-tenth volume of precultured cells $(5 \mathrm{ml})$ was added to $500 \mathrm{ml}$ of fresh Luria-Bertani medium and cultured at $37^{\circ} \mathrm{C}$ until $\mathrm{OD}_{600}$ reached $0.3-0.6$. Protein production was induced by adding isopropyl- $\beta$ - $\mathrm{D}$-thiogalactopyranoside at a final concentration of $0.5 \mathrm{mM}$ and shaking at $37^{\circ} \mathrm{C}$ for $5 \mathrm{~h}$. Cells were collected and resuspended in buffer A (25 mM HEPES, pH 7.5, 20\% glycerol, $1 \mathrm{mM}$ dithiothreitol, $100 \mathrm{mM} \mathrm{NaCl}, 0.2 \mathrm{mM}$ EDTA with protease inhibitor cocktail (Sigma-Aldrich), and $1 \mathrm{mM}$ phenylmethyl sulphonyl fluoride). The cells were lysed using a French press (8500 p.s.i.; one time). The lysates were sonicated for $30 \mathrm{~s}$ twice and centrifuged at $20,000 \mathrm{~g}$ for $20 \mathrm{~min}$. The supernatants were stored at $-80^{\circ} \mathrm{C}$ until use. The IDD $14 \alpha$ and IDD $14 \beta$ cDNAs were amplified by RT-PCR and were subcloned into the pGADT7 vector. The IDD $14 \alpha$ and IDD $14 \beta$ polypeptides were labelled with ${ }^{35} \mathrm{~S}$-Met using the TNT-coupled reticulocyte lysate system (Promega).

The MBP or MBP-IDD14 $\alpha$ proteins were mixed with amylose resin (SigmaAldrich) and agitated for $15 \mathrm{~min}$ at room temperature. The beads were then washed three times with $1 \times$ PBS buffer and one time with buffer A. Five $\mu$ l of the ${ }^{35} \mathrm{~S}$-labelled proteins was added and incubated for $2 \mathrm{~h}$ at $4{ }^{\circ} \mathrm{C}$. The beads were then washed five times with buffer A. The bound proteins were eluted with $1 \times$ SDSloading buffer by boiling for $5 \mathrm{~min}$ at $100^{\circ} \mathrm{C}$ and subject to SDS-polyacrylamide gel electrophoresis (PAGE) and autoradiography.

Interactions between IDD $14 \alpha$ and IDD $14 \beta$ were also examined by immunoprecipitation assays. The IDD14 $\alpha-M Y C$ and IDD $14 \beta-M Y C$ gene fusions, in which a MYC-coding sequence was fused in-frame to the $3^{\prime}$ ends of the IDD14 $\alpha$ and IDD14 $\beta$ genes, were expressed transiently in leaf cells of $N$. benthamiana as described previously ${ }^{4}$. The recombinant MBP-IDD14 $\alpha$ fusion protein was prepared in E. coli cells. Total proteins were extracted from the $N$. benthamiana leaf cells and mixed with the recombinant MBP-IDD $14 \alpha$ protein using extraction buffer $(50 \mathrm{mM}$ HEPES, pH 7.9, 20\% glycerol, $1 \mathrm{mM}$ dithiothreitol, $100 \mathrm{mM} \mathrm{NaCl}$ and $0.2 \mathrm{mM}$ EDTA) containing a protease inhibitor cocktail (Sigma-Aldrich). After incubation at $4{ }^{\circ} \mathrm{C}$ for $6 \mathrm{~h}$, the mixtures were immunoprecipitated using amylose resin. The beads were washed four times with fresh extraction buffer supplemented with $0.1 \%$ Triton X-100, and the bound proteins were eluted with $1 \times$ SDS-PAGE loading buffer. The eluted proteins were subject to SDS-PAGE, and the IDD14 $\alpha$-MYC and IDD14 $\beta$-MYC fusion proteins were detected by western blot analysis using an anti-MYC antibody.

Bimolecular fluorescence complementation assays. BiFC assays were carried out by co-transfection of the IDD14 $\alpha$-nYFP and IDD14 $\beta$-cYFP vectors or vice versa into the Arabidopsis mesophyll protoplasts as described previously ${ }^{26}$. Sixteen hours after transfection, reconstitution of yellow fluorescent protein (YFP) fluorescence was observed using a confocal microscope with the following YFP filter set up: excitation $515 \mathrm{~nm}, 458 / 514$ dichroic and emission 560-615 BP filter.

Measurement of starch contents. For individual assays, $\sim 0.5 \mathrm{~g}$ of aerial plant parts was used to measure contents of soluble sugars using the Sucrose/D-Glucose Assay kit or the Starch Assay kit, according to the manufacturer's protocols (Megazyme). After grinding plant materials in liquid nitrogen, soluble sugars were extracted two times with $80 \%$ ethanol at $70^{\circ} \mathrm{C}$, and fractions were collected. They were then centrifuged at $12,000 \mathrm{~g}$ for $10 \mathrm{~min}$. Ethanol was evaporated from the supernatant using a vacuum evaporator, and the solution volume was adjusted to $400 \mu \mathrm{l}$ by adding triple-distilled water to measure sugar contents. The insoluble pellets were used to measure starch contents. 
Chromatin immunoprecipitation assays. A MYC-coding sequence was fused in-frame to the $3^{\prime}$ ends of the IDD $14 \alpha$ and IDD $14 \beta$ genes, and the fusions were subcloned under the control of the CaMV $35 \mathrm{~S}$ promoter. The expression construct was transformed into Arabidopsis plants. Three-week-old 35S:IDD14 $\alpha-M Y C$ and 35S:IDD14 $\beta-M Y C$ transgenic plants grown on $1 / 2 \times$ Murashige and Skoog agar plates were used for extraction of total cellular extracts. Processing of plant materials and qRT-PCR were carried out as described previously ${ }^{27}$. The qRT-PCR primers used are listed in Supplementary Table S3.

Transcriptional activation activity assays. For transient expression assays in Arabidopsis protoplasts, several reporter and effector plasmids were constructed. The reporter plasmid contains four copies of the GAL4 upstream activation sequence and the $\beta$-glucuronidase (GUS) gene. To construct the p35S:IDD14 effector plasmids, the IDD $14 \alpha$ and IDD $14 \beta$ genes were fused to the GAL4 DNAbinding domain and inserted into an expression vector containing the CaMV $35 \mathrm{~S}$ promoter. The reporter and effector plasmids were cotransformed into Arabidopsis protoplasts by a polyethylene glycol-mediated transformation method. The GUS activities were measured by the fluorometric method as described previously ${ }^{28}$ A CaMV 35S promoter-luciferase (LUC) construct was also cotransformed as an internal control. The luciferase assay was carried using the Luciferase Assay System kit (Promega)

\section{References}

1. Vinson, C., Acharya, A. \& Taparowsky, E. J. Deciphering B-ZIP transcription factor interactions in vitro and in vivo. Biochim. Biophys. Acta 1759, 4-12 (2006).

2. Benezra, R., Davis, R. L., Lockshon, D., Turner, D. L. \& Weintraub, H. The protein Id: a negative regulator of helix-loop-helix DNA binding proteins. Cell 61, 49-59 (1990).

3. Wenkel, S., Emery, J., Hou, B. H., Evans, M. M. \& Barton, M. K. A feedback regulatory module formed by LITTLE ZIPPER and HD-ZIPIII genes. Plant Cell 19, 3379-3390 (2007)

4. Kim, Y. S. et al. HD-ZIP III activity is modulated by competitive inhibitors via a feedback loop in Arabidopsis shoot apical meristem development. Plant Cell 20, 920-933 (2008).

5. $\mathrm{Hu}, \mathrm{W}$. \& $\mathrm{Ma}, \mathrm{H}$. Characterization of a novel putative zinc finger gene MIF1 involvement in multiple hormonal regulation of Arabidopsis development. Plant J. 45, 399-422 (2006).

6. Hong, S. Y. et al. Nuclear import and DNA binding of the ZHD5 transcription factor is modulated by a competitive peptide inhibitor in Arabidopsis. J. Biol. Chem. 286, 1659-1668 (2010).

7. Hyun, Y. \& Lee, I. KIDARI, encoding a non-DNA Binding bHLH protein, represses light signal transduction in Arabidopsis thaliana. Plant Mol. Biol. 61, 283-296 (2006).

8. Macknight, R. et al. Functional significance of the alternative transcript processing of the Arabidopsis floral promoter FCA. Plant Cell 14, 877-888 (2002).

9. Roman, C., Cohn, L. \& Calame, K. A dominant negative form of transcription activator mTFE3 created by differential splicing. Science 254, 94-97 (1991).

10. Kim, H. J. et al. Identification of a truncated alternative splicing variant of human PPARgammal that exhibits dominant negative activity. Biochem. Biophys. Res. Commun. 347, 698-706 (2006).

11. Miura, K. et al. SIZ1-mediated sumoylation of ICE1 controls CBF3/DREB1A expression and freezing tolerance in Arabidopsis. Plant Cell 19, 1403-1414 (2007).

12. Brocard-Gifford, I., Lynch, T. J., Garcia, M. E., Malhotra, B. \& Finkelstein, R. $\mathrm{R}$. The Arabidopsis thaliana ABSCISIC ACID-INSENSITIVE8 encodes a novel protein mediating abscisic acid and sugar responses essential for growth. Plant Cell 16, 406-421 (2004).

13. Gottwald, J. R., Krysan, P. J., Young, J. C., Evert, R. F. \& Sussman, M. R. Genetic evidence for the in planta role of phloem-specific plasma membrane sucrose transporters. Proc. Natl Acad. Sci. USA 97, 13979-13984 (2000).

14. Coneva, V., Zhu, T. \& Colasanti, J. Expression differences between normal and indeterminate1 maize suggest downstream targets of ID1, a floral transition regulator in maize. J. Exp. Bot. 58, 3679-3693 (2007).
15. Tanimoto, M., Tremblay, R. \& Colasanti, J. Altered gravitropic response, amyloplast sedimentation and circumnutation in the Arabidopsis shoot gravitropism 5 mutant are associated with reduced starch levels. Plant Mol. Biol. 67, 57-69 (2008)

16. Li, L. et al. Identification of the novel protein QQS as a component of the starch metabolic network in Arabidopsis leaves. Plant J. 58, 485-498 (2009).

17. Eimert, K., Wang, S. M., Lue, W. I. \& Chen, J. Monogenic recessive mutations causing both late floral initiation and excess starch accumulation in Arabidopsis. Plant Cell 7, 1703-1712 (1995).

18. Yano, R., Nakamura, M., Yoneyama, T. \& Nishida, I. Starch-related alphaglucan/water dikinase is involved in the cold-induced development of freezing tolerance in Arabidopsis. Plant Physiol. 138, 837-846 (2005).

19. Kaplan, F. \& Guy, C. L. RNA interference of Arabidopsis beta-amylase8 prevents maltose accumulation upon cold shock and increases sensitivity of PSII photochemical efficiency to freezing stress. Plant J. 44, 730-743 (2005).

20. Kaplan, F. \& Guy, C. L. Beta-amylase induction and the protective role of maltose during temperature shock. Plant Physiol. 135, 1674-1684 (2004).

21. Lu, Y. \& Sharkey, T. D. The importance of maltose in transitory starch breakdown. Plant Cell Environ. 29, 353-366 (2006).

22. Maruyama, K. et al. Metabolic pathways involved in cold acclimation identified by integrated analysis of metabolites and transcripts regulated by DREB1A and DREB2A. Plant Physiol. 150, 1972-1980 (2009).

23. Udvardi, M. K., Czechowski, T. \& Scheible, W. R. Eleven golden rules of quantitative RT-PCR. Plant Cell 20, 1736-1737 (2008).

24. Kim, Y. S. et al. A membrane-bound NAC transcription factor regulates cell division in Arabidopsis. Plant Cell 18, 3132-3144 (2006)

25. Gutierrez, L. et al. The lack of a systematic validation of reference genes: a serious pitfall undervalued in reverse transcription-polymerase chain reaction (RT-PCR) analysis in plants. Plant Biotechnol. J. 6, 609-618 (2008).

26. Yoo, S. D., Cho, Y. H. \& Sheen, J. Arabidopsis mesophyll protoplasts: a versatile cell system for transient gene expression analysis. Nat. Protoc. 2, 1565-1572 (2007).

27. Lawrence, R. J. et al. A concerted DNA methylation/histone methylation switch regulates rRNA gene dosage control and nucleolar dominance. Mol. Cell 13, 599-609 (2004)

28. Jefferson, R. A., Kavanagh, T. A. \& Bevan, M. W. GUS fusions: betaglucuronidase as a sensitive and versatile gene fusion marker in higher plants. EMBO J. 20, 3901-3907 (1987).

\section{Acknowledgments}

This work was supported by the Leaping Research Program (20100014373) provided by the National Research Foundation of Korea and by grants from the Plant Signaling Network Research Center (20100001457), the National Research Foundation of Korea (20100028147), and from the Agricultural R \& D Promotion Center (309017-05-2-HD130), Korea Ministry for Food, Agriculture, Forestry and Fisheries. P.J.S. was supported by the Seoul Science Fellowship. We thank Emily Wheeler for editorial assistance.

\section{Author contributions}

C.-M.P. conceived and designed the experiments. C.-M.P. wrote the paper with the help of P.J.S. P.J.S., M.J.K., J.-Y.R. and E.-Y.J. conducted the experiments and contributed to the study design.

\section{Additional information}

Supplementary Information accompanies this paper at http://www.nature.com/ naturecommunications

Competing financial interests: The authors declare no competing financial interests.

Reprints and permission information is available online at http://npg.nature.com/ reprintsandpermissions/

How to cite this article: Seo, P. J. et al. Two splice variants of the IDD14 transcription factor competitively form nonfunctional heterodimers, which may regulate starch metabolism. Nat. Commun. 2:303 doi: 10.1038/ncomms1303 (2011). 